\title{
Managing the diabetic foot in resource-poor settings: challenges and solutions
}

This article was published in the following Dove Press journal:

Chronic Wound Care Management and Research

27 October 2017

Number of times this article has been viewed

\author{
Zulfiqarali G Abbas ${ }^{1,2}$ \\ 'Department of Internal Medicine, \\ Muhimbili University of Health and \\ Allied Sciences, Dar es Salaam, \\ Tanzania; ${ }^{2}$ Department of Internal \\ Medicine, Abbas Medical Centre, Dar \\ es Salaam, Tanzania
}

Abstract: Diabetes mellitus is one of the most common noncommunicable diseases globally. In Africa, rates of diabetes are increasing, so there is a parallel increase of foot complications. Peripheral neuropathy is the main risk factor for foot ulceration in people with diabetes in developing nations, but peripheral arterial disease is also increasing in number owing to the change in lifestyle and increasing urbanization. Ulceration arising from peripheral neuropathy, peripheral arterial disease, and trauma are highly susceptible to secondary infection and gangrene, and are hence associated with increased morbidity and mortality. Government funding is very limited in many developing countries, and diabetes and its complications impose a heavy burden on health services. In particular, the outcomes of foot complications are often poor, and this is the result of various factors including lack of awareness of the need for foot care among patients, relatives, and health care providers; relatively few professionals with an interest in the diabetic foot and with the training to provide specialist treatment; nonexistent podiatry services; long distances for patients to travel to the clinic; delays among patients in seeking medical care, or the late referral of patients for specialist opinion; and lack of the awareness of the importance of a team approach to care, and the lack of training programs for health care professionals. Many of these can, however, potentially be tackled without exorbitant spending of financial resources. Cost-effective educational efforts should be targeted at both health care workers and patients. These include implementation of sustainable training programs for health care professionals with a special interest in foot care and focusing to disseminate the foot care information, on the prevention and management of the diabetic foot, to other health care professionals and to patients. The Step by Step foot program is an educational program that was first carried out in Tanzania and India. In conclusion, the only way to move forward in developing countries is educational programs on prevention and management of the diabetic foot. An interdisciplinary team approach is needed, and not multidisciplinary, for the management of the diabetic foot. Patients presenting early at a less severe stage will definitely lead to reduction in morbidity and death rates.

Keywords: Africa, diabetic foot, peripheral neuropathy, peripheral arterial disease, amputation, diabetic foot ulcers, Step by Step foot project

\section{Introduction and epidemiology}

Diabetes mellitus is a serious condition leading to devastating outcomes for patients across the globe. ${ }^{1,2,3}$ It affects all social grades of people from both the developed and the developing world, young or old, male or female, rich or poor.

The global prevalence of diabetes in 2015 was estimated at 415 million. ${ }^{3}$. Due to longer life, changes in dietary patterns, and increasingly sedentary lifestyle, prevalence
Correspondence: Zulfiqarali G Abbas Abbas Medical Center, P.O. Box 21361, Dar es Salaam, Tanzania Tel +25522 2183936 Email zabbas@cats-net.com 
of diabetes is expected to increase to 642 million by $2040 .^{3}$ Africa and Asia are going to be particularly badly affected compared to the rest of the world. For example, the prevalence in Africa is estimated to increase from 14.2 million in 2015 to 34.2 million by $2040 .^{3}$

Africa is currently home to 1.1 billion people, and its population is predicted to reach 2.4 billion by $2050 .{ }^{4}$ It has 54 countries, is home to some 1,000 ethnic groups, and has more than 1,000 languages. ${ }^{1}$ Much of the increase in population will happen in sub-Saharan Africa, which includes many of the poorest countries. Similar to other parts of the world, as the population grows, more people in sub-Saharan Africa will live in cities, consume more processed food, and develop obesity, heart disease, cancer, and type 2 diabetes. ${ }^{4}$

Diabetes is the main cause of morbidity and mortality in both developed and developing countries. ${ }^{5-17}$ Some complications of diabetes include blindness, renal failure, and cardiovascular complications, but complications connected to the foot are related to the highest rates of morbidity and mortality. ${ }^{5-17}$ Complications of the foot like septic foot can be very serious and extremely costly. ${ }^{5-21}$ Among all the lower limb nontraumatic amputations performed across the globe, $40 \%-60 \%$ are performed in patients with diabetes. ${ }^{18}$ Globally, one million amputations are performed annually on people with diabetes. ${ }^{18}$ In 2005, it was estimated that every $30 \mathrm{sec}-$ onds a limb is lost due to diabetes somewhere in the world. ${ }^{18}$ After 10 years, in 2015, it was again estimated that every 20 seconds a limb is amputated due to diabetes somewhere in the world. ${ }^{18}$ In the time period of one decade, things are getting worse rather than improving.

\section{Financial burden of diabetic foot diseases}

The cost of treating the diabetic foot in the Western world is already available in the literature. There are few studies of the cost of treating the diabetic foot in the developing world, although one recent study compared the cost between five countries from three continents. ${ }^{19}$ All five countries were presented with a hypothetical case from which to estimate cost of care. It was estimated that it would cost $\$ 3,060$ in Tanzania, which is only $1.6 \%$ of the cost of treating the same ulcer in the USA $(\$ 188,645) .{ }^{19}$. Even today in most of the African countries, medical expenses are paid by the family, and only now in some of the countries has medical insurance started operating and paying medical expenses. It is devastating for the patient if he is the only bread earner in an extended family, which is common in Africa. ${ }^{8-11,19}$ The cost estimated was approximately 2 years of average income for the patients in Tanzania. ${ }^{19}$ During the period of management of foot ulcer, the patient is not able to work, and so loss of productivity is again an added cost to the family, relatives, friends, and community. ${ }^{19}$

\section{The extent of diabetic foot risk in developing countries}

The greatest factors underlying risks of diabetic foot disease are peripheral neuropathy $(\mathrm{PN})$ and peripheral arterial disease (PAD). PN exposes the foot to risk of unnoticed trauma. PAD limits the capacity for healing, especially when the wound is worsened by infection.

\section{$\mathrm{PN}$}

Globally, PN is the most common complication of diabetes. If one looks at the literature, $\mathrm{PN}$ in the Western world is seen in $5 \%-80 \%$ of patients with diabetes. ${ }^{20-21}$ The same applies to African countries if you look at the prevalence rates of PN. ${ }^{1-3,5-11,13-16}$ In Tanzania, one of the studies showed symptomatic PN in patients attending diabetic clinics was estimated to be $25 \% .^{13}$ Similarly, published reports of PN across Africa showed that in 1960s, PN rate in Zimbabwe was $4 \%,{ }^{22}$ in $1970 \mathrm{~s}$ in Ethiopia it was $40 \%,{ }^{23}$ in $1980 \mathrm{~s}$ in Ethiopia it was $47 \%,{ }^{24}$ in 1990 s in Nigeria it was $68 \%,{ }^{25}$ and in 2000 in Algeria it was $80 \%{ }^{26}$ Two multicentre studies have shown that there is no difference in prevalence based on race either between countries and continents or within them. ${ }^{14-15}$

\section{PAD}

Another important complication of diabetic foot is PAD, which causes high morbidity and mortality in patients with diabetes. It is very commonly seen in the Western world, but it was not that common in Africa and Asia. Time has changed the situation across Africa and Asia now, and rates of PAD are increasing. The reason for this is that communities in Africa and Asia are becoming more urbanized, following a sedentary lifestyle, lacking exercise, changing diet, etc. As a consequence, PAD is increasing in people with diabetes. ${ }^{1,2,13,14,26,27,28}$ One review published literature from the African continent and the trend of PAD rates have increased over the years as follows, from as low as $0 \%$ from Zimbabwe in $1960 \mathrm{~s},{ }^{22} 1.1 \%$ from Ethiopia in $1970 \mathrm{~s},{ }^{23}$ and $2.9 \%$ from Tanzania in $1980 \mathrm{~s},{ }^{29}$ to high as $54 \%$ from Nigeria in $1990 \mathrm{~s},{ }^{25}$ and $84.4 \%$ from Algeria of North Africa in $2000 .{ }^{26}$ The reality is that PAD rates are similar in Africa as seen in the Western world. Urban populations from Tanzania showed the same high rates of $26 \%$ in $2009 .{ }^{14}$

\section{Diabetic foot ulcer and amputation}

The reality in Africa is that high rates of diabetic foot ulcers, amputation, and mortality are observed all across the 
continent. In 2002, Tanzania had $15 \%$ of diabetes patients admitted because of foot ulcers, and 33\% of those underwent amputation, and mortality rates were very high; $54 \%$ in those patients who presented with extreme pathology (Wagner score $\geq 4) .{ }^{11}$ This is also true for other parts of Africa as well, as they show high rates of morbidity and mortality. ${ }^{1,2,5,7,9,11,22-32}$

\section{Diabetic foot care education}

Education is the most important tool in Africa to prevent diabetic foot ulcers. At the primary health care center, or district health care center, or even in some regional health care centers, people with diabetes lack foot care education..$^{9,10}$ Due to the lack of basic education that an ulcer can lead to an infected diabetic foot ulcer, patients present with acute limb-threatening conditions, leading to amputation in about $25 \%-50 \%$ of the people. ${ }^{18}$ Educational programs are required at the grass root level to prevent complications of foot ulcers in the developing world. ${ }^{19,33-40}$ The benefit of improved foot care education has been clearly demonstrated. ${ }^{34-40}$ More widespread implementation would lead to improved clinical outcome at reduced cost.

\section{What are the challenges and solutions?}

The challenge posed by the diabetic foot is a particular problem in the developing world, and proportionately more so than in more affluent countries. In the past $>20$ years, developed nations have increased research on molecular epidemiology and genetics. This escalation of research in genetics has actually not benefited patient care and outcome of the patients in countries where routine diabetes care is required.

Financial resources allocated in Europe and North America are offset by increased burden of diabetes and its limb complications, and ready availability of technology, drugs, and antimicrobials. In North America, the high cost of health care and insurance premiums often precludes optimal provision of diabetic foot care. The only research in the area of epidemiology done in developing nations, including from the African continent, that has had any significant impact on patient outcomes has been characterization of diabetic complications, especially of the lower limb, and implementation of the findings to evidence-based practice. Neuropathy and infection play a major role, and the affected population is generally younger. When, however, this is associated with the rising incidence of PAD, the health care systems in continents such as Africa could become overwhelmed. 1,2,8,12,24,41-44

The solution to the major challenges is how to implement the current epidemiologic realities and programs that could truly improve the outcomes of diabetic foot complications, using relatively small proportions of available resources. The challenge is to find a solution to the problem posed by diabetic foot disease in resource-poor countries.

\section{Managing infection in the diabetic foot}

Almost all diabetic foot ulcers in Africa are infected at the time of first assessment. ${ }^{1,2,5-11,25,28,33,41,45-46}$ The reason is that patients typically present too late to health centers, and so the patient has either developed gangrene or limb-threatening infection. ${ }^{11}$.

In most of the resource-poor countries, where there are no podiatrist or chiropody services, diabetic foot infections are very common due to several reasons and many lesions initially are ignored or noticed relatively late or after unsuccessful home treatment such as bathroom surgery, soaking in water, application of home remedies, going to a faith healer, or applying herbal medication. It is for such reasons that many people may only seek medical help when either serious infection or gangrene supervenes. The later an ulcer presents, the less likely it is that the foot can be saved. ${ }^{11,18,28}$ Outcomes of foot complications are preventable and can be reduced through proper foot care. ${ }^{28}$

The least invasive foot infection is cellulitis, a deep soft tissue infection that involves fascia, muscles, and deep tissue, and osteomyelitis, when bone is involved. ${ }^{11,18,28}$ Chronic foot ulcers lead to contiguous spread of soft tissue infection in an ulcer, to the underlying bone. ${ }^{47}$ Bone infection (osteomyelitis) can be diagnosed by simple clinical methods - probing to the bone beneath an ulcer with a sterile blunt instrument, with or without bone radiography, isotope bone scans, or histology. As nuclear medicine services and histology tests may not be routinely available at centers in developing countries, greater reliance has to be placed on clinical signs, and these are not $100 \%$ reliable. Abbas et al usually use the simple bedside test of physically probing the bone beneath an ulcer to diagnose the presence or absence of osteomyelitis. $5,6,9,10,28$ If osteomyelitis is diagnosed, then the necrotic tissue is removed until viable tissue is visible and this is followed by treatment with antibiotics. If in any case foot ulcer prognosis looks poor, then the patient is referred for surgical assessment with a view to remove necrotic bone or to undergo amputation if necessary.

Infection is diagnosed purely on clinical grounds, and the role of microbiological sampling is restricted to the identification of probable pathogens and the sensitivity to antibiotics. Superficial swab specimens for culture and sensitivity are not very useful due to polymicrobial growth, which is largely uninterpretable. ${ }^{18,33}$ Deep tissue biopsy is likely to give more useful information. However many microbiology services 
in resource-poor countries do not have facilities to analyze biopsy specimens on a routine basis.

For this reason, Abbas et $\mathrm{al}^{33}$ had to look for solutions to overcome this in less-developed countries by conducting epidemiologic and microbiologic studies and by the use of antibiotics. Also, the use of Gram staining to observe microorganisms can be done as a bedside test at any clinic in the countries with poor resources. We looked at the utility of Gram stains in comparison with culture and sensitivity in the management of limb ulcers in people with diabetes. We found a sensitivity of more than $90 \% .{ }^{33}$ We found that clinical outcome following use of empirically chosen broad-spectrum antimicrobials (without microbiology cultures), in conjunction with surgical debridement, yielded similar or better outcomes than antimicrobials chosen by antimicrobial susceptibility testing of pathogens isolated from deep tissue cultures. ${ }^{18,33}$

The limited availability of different antibiotic preparations, as well as the cost of some of them, is a major problem in less-developed countries and contributes to the relatively poor outcome of infected ulcers and further emphasizes the need for early expert assessment.

Utilization of cheap broad-spectrum antibiotics has to be sourced from India, as suggested by Abbas et al, ${ }^{6}$ as these are similar or better than the more expensive antimicrobials obtained from the West. On the other hand, the widespread use of broad-spectrum preparations carries the risk of worsening the threat of increasing antimicrobial resistance.

\section{Challenges and solutions by using a monitoring system}

A monitoring system requires huge amounts of funding and human resources for data collection, statistics analysis of data, etc. The challenges that Abbas et al face in the management of the diabetic foot are the institution and maintenance of a monitoring system. In countries with poor resources, it is important to establish a monitoring system for diabetic limb complications, for a number of reasons: 1) to screen risk factors (PN and PAD) through monitoring data; 2) to be able to take proper active and preventive responses to selected high-risk diabetes populations; and 3) to intervene effectively. Abbas et al have instituted perhaps the largest monitoring system for limb complications in Africa, in Tanzania. ${ }^{1,2,3,5-11,13-16}$ The monitoring system has been sustained through various reasons like strict case definition of limb complications, good quality questionnaires used, and the commitment of the staff also trained in collection of data and utilization of these data for evidence-based practice. By maintaining the system in Tanzania, significant and positive effects on patients were observed, as their medical condition and how to deal with diabetic foot complications was known. Due to this system, patients also become knowledgeable about diabetes and its complications. They keep their appointments to attend the clinics, and have to even come if the clinics are far from their home.

\section{The importance of district/regional hospitals}

Regional or district hospitals are usually large centers in the developing countries under the control of the Ministry of Health. They can provide medical, surgical, and basic laboratory services for every region, even though resources can be restricted. This setup is found mostly in the developing world such as in Southeast Asia, Africa, Latin America, and the Caribbean and can be centralized for optimizing the use of resources. Regional hospitals are big centers in particular regions of the country that are the teaching centers for medicine in that area. They commonly have specialized medical, surgical, and laboratory (including microbiology) units and often work as reference centers. Agencies such as World Health Organization, United States Agency for International Development, and the UK Department for International Development would generally prefer to maintain collaboration through these centers because of their better infrastructure, trained personnel, and access to the Ministry of Health.

\section{Time is tissue - decision to seek medical assistance quickly}

It is widely known that time is very important in myocardial infarctions and cerebral vascular accidents. What is not well known is that a patient with a diabetic foot ulcer, days, weeks or a month may mean the difference in saving a toe, foot, leg, or even life. These patients cannot afford to present late to a health center. If the patient has an underlying infection and/ or ischemia it is even more critical that the treatment begins early. Time is tissue in diabetic foot ulcers. ${ }^{11,28}$ Reasons for delayed presentations were found by Abbas et al, and most of the time these were associated with cultural, traditional, and customary behaviors. Patients in developing countries have a tendency to go through several stages before reporting to the health center. They will first try to attempt to treat small lesions like a callus or a boil at home using a razor blade or herbal solution. These procedures are carried out by the patient themselves or relatives. If home treatment fails, the patient's next move would be to see a faith healer or herbal doctor. People in villages and towns usually have very high respect and faith in the faith healer. Herbal healers are seen at almost every corner of the city in Africa, advertising and 
claiming that they can cure all diabetic complications. When herbal medications given by herbalists fail, patients decide to visit the primary health center or district or regional hospitals, where no diabetic foot specialists are found. All such delays can mean that the condition of the foot deteriorates, and so by the time they present to a specialist service, it may be too late to save the foot, and sometimes even to prevent death from severe sepsis. ${ }^{11,28,46}$ Patients who believe strongly in cultural traditions will not get proper and accurate treatment of diabetic foot, and this in turn leads to amputation or death as an outcome. ${ }^{28}$ Some patients delay in getting expert opinion because of the fear of losing a limb, as, in Africa, loss of limb is considered a worse outcome that having the disease. Lack of knowledge among health care personnel at the grass root level can also lead to poor outcomes.

It is commonplace for surgeons in particular to recommend soaking of the foot which has an ulcer, but this is counterproductive as soaking can result in maceration of the skin and hence predispose it to infection (especially by Pseudomonas species which prefer a damp environment).

Many people with diabetes are unaware of the need for early expert referral, and this lack of awareness is obviously made worse by neuropathy with reduced pain sensation. Patients with severe loss of sensation can sustain unnoticed injuries and even rat bites when in bed at night. ${ }^{16}$ Many patients have not been taught to examine their feet on a daily basis to detect cracks or injury. ${ }^{16}$ In contrast, those with symptomatic (painful) $\mathrm{PN}$ - which is a common complication among both educated and less well educated people with diabetes - generally seek medical help faster and so there is no delayed detection of injuries, leading to better outcomes. ${ }^{1,2,3,5-11,13-16}$

\section{The need for specifically designed structures of care} Multidisciplinary working: It is a key recommendation of care in developed countries that professionals should work in close collaboration with colleagues with different specialty training. Physicians should work closely with surgeons, and both should work closely with other specialists, including nurses, orthotist (a person who is qualified to practice orthotics) /pedorthists/shoe fitters, and most important of all - podiatrists. In countries that routinely employ podiatrists, they rapidly become a central member of the team.

In creating a template for optimal care, however, it is important to remember that the team includes not just those who work in a secondary or tertiary referral center but also those in the community. Indeed, the team includes everyone to whom the person with diabetic foot disease turns to for help - including those in primary care as well as nonprofessionals. The primary aim of everyone on this team should be to ensure that every new case of ulceration is reviewed by someone with expert knowledge as soon as possible.

\section{The need for specific emphasis on education}

Education is the only tool we have in the developing world which is free to the patient, and it can be effective if implemented effectively. Such education falls into two categories: education related to prevention and related to treatment. It also needs to be directed at two separate groups: people with diabetes and professionals.

\section{Prevention}

An educational program for foot ulcer prevention should be targeted at health care workers, and it will then become their responsibility to pass on appropriate advice to their patients. Professionals need to learn the five main pillars of preventive diabetic foot, which are as follow: 1) Regular inspection and examination of the feet and footwear, 2) identification of high-risk patients, 3 ) education of the high-risk patients, relatives, friends, and health care workers, 4) appropriate footwear for diabetic patients, 5) treatment of nonulcerative pathology. By implementing these five pillars, limb amputation can be reduced by more than $50 \%$. Several successful preventive educational programs have been carried out in developed and developing countries. ${ }^{34-37,38-40,48-50}$

\section{Step by Step foot project}

It was for these reasons that we adopted the Step by Step foot project in Tanzania as a way to improve educational skills as well as to improve the management of diabetic foot problems. ${ }^{35}$ The Step by Step project focused on all the aspects of diabetic foot care and was initiated in collaboration with the International Working Group of Diabetic Foot, now called D-Foot International, Diabetic Foot Society of India, and Muhimbili University of Health and Allied Sciences, Dar es Salaam.

The main objectives of the Step by Step project were as follows: 1) To create more awareness of diabetic foot problems among health care workers and patients, 2) to provide sustainable training of health care professionals in diabetic foot management, 3) to facilitate the transfer of information and expertise among health professionals and export ideas to other developing countries, 4) to reduce the risk of lower limb complications in people with diabetes, and 5) to empower people with diabetes to care for their feet better, detect problems earlier, and seek timely help when problems 
arise. ${ }^{35}$ This program showed a greater than $50 \%$ reduction in amputation rates. ${ }^{35}$ It was recorded that ulceration, infection, and amputation were prevented through this organized Step by Step foot project, which included a preventive and therapeutic approach to save the diabetic limb. ${ }^{35}$ It is extremely important and mandatory that health care workers who are taking care of patients with diabetes should be able to conduct the simple screening for PN, PAD, and the musculoskeletal system to identify patients with high risk of getting foot infections. An educational program should be the responsibility of the caregiver, and it should be structured to make sure that people with diabetes receive most of the information. Abbas et al ${ }^{35}$ targeted rural health centers dealing with diabetes in Tanzania - the first people to see the foot complications. The Step by Step project was started in these centers, and the health workers there were largely untrained professionals. ${ }^{35}$ When the project was started, it was not surprising that, as with many developing countries, we had a lack of maintenance of infrastructure for diabetic foot management, lack of trained personnel, no podiatry services, and no structural educational curriculum established on the diabetic foot for training health care workers in the country. There was no concept of a superspecialty, such as diabetic foot, to support training of the health care worker who helps people with diabetes. This was the reason the Step by Step foot project was designed with an aim to educate these health care workers at the grass roots level and was instituted in several regions across Tanzania. ${ }^{35}$

The Step by Step project was a training program primarily focusing on all the aspects of diabetic foot. The program consisted of two consecutive education seminars given a year apart, each event lasting three days. All the participants were given educational material and a screening, diagnostic, and therapeutic basic kit of instruments focused on diabetic foot at the time of registration for the first basic course. ${ }^{34-37}$ These materials included written, visual, and audiovisual information all directed to diabetic foot complications. Posters were also given for the clinics on the topic of diabetic foot. These educational materials were aimed at both patients and health care workers (doctors and nurses). ${ }^{34-37}$ The entire Step by Step foot project curriculum focused on all the aspects of diabetic foot and included formal lectures, workshops, practical demonstration with live patients, and hands-on experience training. ${ }^{34-37}$ On completion of the course, all the delegates are expected to disseminate the knowledge to fellow colleagues and conduct the course at their centers. ${ }^{34-37}$ It is practical in the developing world, when the health care worker has to see lots of patients in a day, to divide patients according to the risk factors. Patients with relatively low risk or normal diabetes foot receive normal general education once a year, and this should be enough. On the other hand, patients with a high-risk foot (PN, PAD, neuroischemic foot, previous ulcers, or amputations) should receive intensive education on the care, prevention, and therapeutic aspects of diabetic foot. ${ }^{34-40,49-50}$ Basic and advanced foot care education is given to all health care providers. For patients with disability who cannot look after themselves, special education is given to their assistants and care providers. ${ }^{34-40,49-50}$

Since the introduction of the Step by Step diabetic foot training program in Tanzania, the 2004 basic course and the 2005 advanced course have improved outcomes of the diabetic foot ulcers, more ulcers are reported at the rural health center at early stages, more awareness has been created among the patients with diabetes, less patients are now referred to tertiary care centers in the country, and less amputations are reported. ${ }^{35}$ On the other hand, it has also created more awareness among health care workers and patients, and so more training is conducted among health care workers. ${ }^{35}$

As the pilot course of Step by Step foot project showed success in Tanzania from 2004 and 2005, further training was held in a further 30 private health centers, including 30 teams of medical officers and nurses, leading to a total of 60 participants. The same course of the basic and advanced lessons was conducted. ${ }^{35}$ Cobblers were invited from respected centers in 2010 to extend the multidisciplinary approach so as to make a good training team with medical workers. ${ }^{35}$

This was followed by inviting surgeons from the same centers where medical officers and nurses were trained and from institutes that have surgical facilities. In 2009, the first Step by Step diabetic foot surgical training project specifically aimed at surgeons from selected centers in 20 regions was held in Tanzania; the focus was largely on optimizing surgical practice to salvage diabetic feet and reduce rates of amputation. ${ }^{35}$

After successful training projects done in Tanzania and India (which included delegates from India, Bangladesh and Nepal) led to increasing demand for Step by Step foot program to be rolled out in other countries. ${ }^{34-40}$ One of the aims was to export the idea to other nations, and to date the model has been exported to various other countries in Africa (Democratic Republic of Congo, Guinea, Botswana, Malawi, Kenya, Ethiopia, Egypt, Zimbabwe, Mali and recently conducted in Nigeria in October 2017), as well as in Pakistan, and the Caribbean (Barbados, St Lucia, St Maarten, St Kitts, and the British Virgin Islands). ${ }^{38-40}$ 


\section{Train the trainer foot course}

In December 2012, a decision was made to replace the Step by Step foot care project with a Train the Trainer course. The Train the Trainer course targets health workers from different countries in a particular region to come under one roof and disseminate the knowledge once they go back to their own countries. The first successful Train the Trainer course was conducted in Brazil in 2012 with participants from 14 countries in South America. ${ }^{38-40,51}$ In July 2013, the second course was conducted in Tobago (Caribbean) with 57 participants from 22 countries. In February 2015 the third Train the Trainer course was conducted in Bled, Slovenia (Central and Eastern Europe), where 17 Eastern European countries participated. In November 2016, the fourth Train the Trainer course was conducted in Bangkok (Western Pacific region) and helped train health care workers from 13 countries. Train the Trainer courses are being considered in future for African, Asian, and North American countries as well. ${ }^{38-40,51}$

This is the only unique project which has actually started in the developing world (Dar es Salaam, Tanzania) and been ultimately exported to the developed world.

In the author's opinion, improvement in the field of preventive foot care and reducing amputation will only be achieved by focusing on education. Education is the only powerful tool that can work all over the world. The education of health care workers who would in turn educate other colleagues in the center and surrounding areas of the health centers has a cascading effect. These health care workers in the periphery of each country would in turn educate patients, families of the patients, relatives, and friends.

\section{Conclusion}

In summary, the many reasons for poor foot care in various less-developed countries have been discussed at length in this manuscript. While improvements in technology are awaited, the priority is to establish basic principles of training and management in this neglected specialty of diabetes. This can be achieved at minimal cost, and the potential savings, both clinical and financial, are enormous. The key to improvement is the education and empowerment of professionals and, through them, the educational empowerment of people with diabetes. Over the last 20 years, developed countries have demonstrated a considerable reduction in the numbers of major amputations being undertaken for diabetes. The goal is to achieve similar improvements in developing nations, and it is of even greater importance to minimize the enormous increase in this terrible clinical problem that is surely going to accompany the progressive changes of lifestyle that will inevitably occur in the next 20 years.

\section{Acknowledgments}

The author acknowledges and appreciates the members of Step by Step foot project, D-Foot International and Train the Trainer foot course: Kristien Van Acker, Stephan Morbach, Neil Baker, Vilma Urbancic-Rovan, Shigeo Kono, Mounia Sabasse, Nalini Campillo, Karel Bakker, late Ali Foster, Arun Bal, Sharad Pendsey, and Vijay Vishwanathan. He is also thankful for all the helpful discussion with many colleagues during the writing of this manuscript, and in particular to William Jeffcoate and Lennox Archibald. The author thanks Shabneez Gangji and other staff in Dar es Salaam for their assistance.

\section{Disclosure}

The author has no financial involvement with any organization or financial conflict with the subject matter or material discussed in the manuscript. The author reports no other conflicts of interest in this work.

\section{References}

1. Abbas ZG. The Global Burden of Diabetic Foot. In: Sharad Pendsey, editor. Contemporary Management of Diabetic Foot. 1st ed. JP Medical, Ltd; 2013:24-30.

2. Abbas ZG, Archibald LK. Recent International Development: Africa. In: Boulton AJM, Cavanagh P, Rayman G, editor. The Foot in Diabetes. 4th ed. Hoboken, NJ: John Wiley \& Sons Ltd; 2006;379-385.

3. International Diabetes Federation, Diabetes Atlas, Sixth edition, 2015. Available from: http://www.diabetesatlas.org/resources/2015-atlas.html. Accessed October 14, 2017.

4. Atun R, Gale EAM. The challenge of diabetes in sub-Saharan Africa, Vol. 3. Published Online July 20, 2015.

5. Abbas ZG. Diabetic foot - An African Perspective. JSM Foot Ankle. 2016;1(1):1005

6. Abbas ZG, Archibald LK. Challenges for management of the diabetic foot in Africa: doing more with less. Int Wound J. 2007;(4) 305-313.

7. Abbas ZG, Archibald LK. Epidemiology of the diabetic foot in Africa Med Sci Monit. 2005;11(8):RA262-270.

8. Abbas ZG, Viswanathan V. The diabetic foot in Africa and India. Int Diab Monitor. 2007;19:8-12.

9. Abbas ZG, Gill GV, Archibald LK. The epidemiology of diabetic limb sepsis: an African perspective. Diabet Med. 2002;19(11):895-899.

10. Abbas ZG, Archibald LK. The diabetic foot in Sub-Saharan Africa: a new management paradigm. Diab Foot J. 2007;10(3):128-136.

11. Abbas ZG, Lutale JK, Morbach S, Archibald LK. Clinical outcome of diabetic patients hospitalized with foot ulcers, Dar es Salaam, Tanzania. Diabet Med. 2002;19(7):575-579.

12. Tesfaye S, Gill G. Chronic diabetic complications in Africa. African J Diab Med. 2011;19(1):4-8.

13. Abbas ZG, Archibald LK. Foot complications in diabetic patients with symptomatic peripheral neuropathy in Dar es Salaam, Tanzania. Diabetes Int. 2000;10:52-56.

14. Abbas ZG, Lutale JK, Archibald LK. Diabetic foot ulcers and ethinicity in Tanzania: a contrast between African and Asian populations. Int Wound J. 2009;6(2):124-131.

15. Morbach S, Lutale JK, Viswanathan V, et al. Regional difference in risk factors and clinical presentation of diabetic foot lesions. Diabet Med. 2004;21:91-95.

16. Abbas ZG, Lutale JK, Archibald LK. Rodent bites on the feet of diabetic patients in Tanzania. Diab Med. 2005;(22):631-633. 
17. Hennis AJ, Fraser HS, Jonnalogadda R, et al. Explanation for the high risk of diabetes related amputation of black African descent and potential for prevention. Diab Care. 2004;(27):2636-2641.

18. International Working group on the Diabetic Foot (IWGDF). The development of global consensus guidelines on the management and prevention of the Diabetic Foot; 2015. Available from: www.iwgdf.org. Accessed October 14, 2017.

19. Cavanagh P, Attinger C, Abbas ZG, Bal A, Rojas N, Xu ZR. Cost of treating diabetic foot ulcers in five different countries. Diabetes Metab Res Rev. 2012;28(Suppl 1):107-111.

20. Edmonds M, Boulton A, Buckenham T, et al. Report of the diabetic foot and amputation group. Diabet Med. 1996;13:S27-S42.

21. Jeffcoat WJ, Harding KG. Diabetic foot ulcers. Lancet. 2003;361: $1545-1551$.

22. Gelfand M, Forbes JI. Diabetes mellitus in the Rhodesian Africa. $S A f r$ Med J. 1963;37:1208-1213.

23. Belcher DW. Diabetes mellitus in Northern Ethiopia. Ethiop Med J. 1970;8:73-84.

24. Lester FT. Long-standing diabetes mellitus in Ethiopia: a survey of 105 patients. Diabetologia. 1983;25(3):222-225.

25. Akanji AO, Adetuyidi A. The pattern of presentation of foot lesions in Nigerian diabetic patients. West Afr J Med. 1990;9(1):1-5.

26. Benotmane A, Mohammedi F, Ayad F, Kadi K, Azzouz A. Service d'Endocrinologie-Diabetologie, Centre Hospitalo-Universitaire, Oran, Algerie. Diabetic foot lesions: etiologic and prognostic factors. Diabetes Metab. 2000;26(2):113-117.

27. Okello S, Millard A, Owori R, et al. Prevalence of lower extremity Peripheral artery disease among adult diabetes patients in Southwestern Uganda. BMC Cardiovasc Disord. 2014;14:75.

28. Abbas ZG (editor). Pocket Guideline of Diabetic Foot. 1st ed. The SUN Pharma, zabbas@hotmail.com, P. O. Box 21361, Dar es Salaam, Tanzania; 2014.

29. Mhando PA, Yudkin JS. The pattern of diabetic complications in African patients in Dar es Salaam. Trop Geogr Med. 1980;32:317-323.

30. Lester FT. Department of Medicine, Yekatit 12 Hospital, Addis Abeba. Amputations in patients attending a diabetic clinic in Addis Abeba, Ethiopia. Ethiop Med J. 1995;33(1):15-20.

31. Muyembe VM, Muhinga MN. Department of Surgery, Central Provincial Hospital, Nyeri, Kenya. Major limb amputation at a provincial general hospital in Kenya. East Afr Med J. 1999;76(3): $163-166$.

32. Sano D, Tieno H, Drabo Y, Sanou A. Service de Chirurgie Generale et Digestive, CHU de Ouagadougou. Management of the diabetic foot, apropos of 42 cases at the Ougadougou University Hospital Center. Dakar Med. 1998;43(1):109-113.

33. Abbas ZG, Lutale JK, Ilondo MM, Archibald LK. The utility of Gram stains and culture in the management of limb ulcers in persons with diabetes. Int Wound J. 2012;9(6):677-682.
34. Abbas Z, Morbach S. Diabetes foot damage in developing countries: the urgent need for education. Diabetes Voice. 2005;50:15-17.

35. Abbas ZG, Lutale JK, Bakker K, Baker N, Archibald LK. The 'Step by Step' Diabetic Foot Project in Tanzania: a model for improving patient outcomes in less-developed countries. Int Wound J. 2011;8: 169-175.

36. Bakker K, Abbas ZG, Pendsey S. Step by step, improving diabetic foot care in the developing world. A pilot study for India, Bangladesh, The "Step by Step" Diabetic Foot Project in Tanzania, 2004-2010 Sri Lanka and Tanzania. Pract Diab Intern. 2006;23:365-369.

37. Pendsey S, Abbas ZG. The Step-by-Step program for reducing diabetic foot problems: a model for the developing world. Curr Diab Rep. 2007;7(6):425-428.

38. Abbas ZG. Preventing foot care and reducing amputation: a step in right direction for diabetes care. Diab Manag. 2013;3(5):427-435.

39. Abbas ZG. Reducing diabetic limb amputations in developing countries. Expert Rev Endocrinol Metabolism. 2015;10(4):425-434.

40. Abbas ZG. The Diabetic Foot. In: Hinchliffe RJ, Schaper NC, Thompson MM, Tripathi RK, Timaran CH, editors. Preventive Foot Care Programs. 1st ed. JP Medical Ltd; 2014:24-30. Available from: https://www.amazon.co.uk/Diabetic-Foot-Robert-Hinchliffe/dp/1907816623. Accessed September 24, 2017.

41. McLarty DG, Pollitt C, Swai AB. Diabetes in Africa. Diabet Med. 1990;7:670-684.

42. Boulton AJ, Vileikyte L, Ragnarson-Tennvall G, Apelqvist J. The global burden of diabetic foot disease. Lancet. 2005;366:1719-1724.

43. van Houtum WH. Barriers to the delivery of diabetic foot care. Lancet. 2005;366:1678-1679.

44. Ramachandran A. Specific problems of the diabetic foot in developing countries. Diabetes Metab Res Rev. 2004;20(Suppl 1):S19-S22.

45. Akanji AO, Bella AF, Agbedana EO, Osotimehin BO, Adetuyibi A. Risk factors for the development of foot lesions in Nigerian patients with diabetes mellitus. East Afr Med J. 1988;65:602-608.

46. Akanji AO, Famuyiwa OO, Adetuyibi A. Factors influencing the outcome of treatment of foot lesions in Nigerian patients with diabetes mellitus. Q J Med. 1989;73:1005-1014.

47. Lipsky BA. A current approach to diabetic foot infections. Curr Infect Dis Rep. 1999;1(3):253-260.

48. Bakker K, Acker KV, Morbach S, Perry A. Promoting foot care education in developing countries: the Caribbean Diabetic Foot Care Program. Diab Voice. 2009;54(3):15-18.

49. Leese G, Stang D. Strategies for improving diabetic foot care: An example from Scotland. Diab Foot Canada. 2014;2(1):41-45.

50. Van Rensburg GJ. Preventive foot care in people with diabetes: Quality patient education. JEMDSA. 2009;14:1-2.

51. Baker N, Van Acker K, Urbancic-Rovan V, Abbas ZG, Morbach S. The worldwide implementation of the 'Train the Foot Trainer' programme. The Diabetic Foot Journal. 2017;20(2):71-6.
Chronic Wound Care Management and Research

\section{Publish your work in this journal}

Chronic Wound Care Management and Research is an international, peer reviewed, open access, online journal publishing original research, reviews, editorials, and commentaries on the causes and management of chronic wounds and the major issues related to chronic wound management. Topics also include chronic wounds as comorbidities to other

\section{Dovepress}

conditions, patient adherence to therapy, and the economic burden of chronic wounds. The manuscript management system is completely online and includes a very quick and fair peer review system, which is all easy to use. Visit http://www.dovepress.com/testimonials.php to read real quotes from published authors. 